\title{
THE EVALUATION OF MICROFABRICATED GAS FLOW SENSOR BY INFRARED THERMOGRAPHY
}

\author{
Young-Jun Lee ${ }^{\dagger}$, Jong-Gyu Kim, Sung-Hoon Jung, Joo-Hyung Kim* \\ Department of Mechanical Engineering, Inha University, Inha-ro 100, Nam-gu, Incheon 22212, Republic of Korea \\ †Presenting Author: ji2092@inha.edu \\ *Corresponding Author: joohyung.kim@inha.ac.kr
}

\begin{abstract}
We designed and fabricated the gas flow sensor on Si wafer based on the heat dissipation behavior. To analyze the overall phenomena of heat flux dissipation as well as distortion in flow sensor using infrared thermography, simulation model of flow sensor was developed and compared with experimental data. The flow sensor consists of a heater and temperature sensing parts on $\mathrm{Si}_{3} \mathrm{~N}_{4}$ membrane. The thin insulating layer $\mathrm{Si}_{3} \mathrm{~N}_{4}$ deposited by LPCVD (low pressure chemical vapor deposition) on silicon wafer. The heater and sensing layers were fabricated on thin membrane structure by deposited Ti/Pt layer process. From the micro-size heater located in center, different distances of temperature sensor as a sensing layer of re-distribution of heat dissipation pattern by different flow rates were designed to measure flow rates. Therefore, we observed temperature distribution over a flow sensor for several flow velocities.
\end{abstract}

KEYWORDS: Infrared thermography, MEMS, Gas flow sensor, Simulation, Heat dissipation.

\section{INTRODUCTION}

The flow sensors are widely used in various fields such as automobile, mining, agriculture, building services, industrial estate and everyday life. The flow sensor measures the flow rate by measuring heat and differential pressure, temperature, dynamic pressure, number of revolutions, ultrasonic wave propagation time, induced electricity, displacement, occurrence frequency of vortex, force, and so on. On the other hand, Types of flow sensors include a cantilever type thermocouple, a micro bridge type heat resistor, a deformed heat resistor, a silicon channel type heat resistor, a diaphragm type heat resistor, and a heat generating resistor. [1-3] Among them, the flow sensor using the heating resistor can be manufactured more easily than the hot-wire flow meter, and the price, size, strength and response time are much superior. In this study, We fabricated a flow sensor composed of a platinum heater and temperature sensor propose a high-precision flow sensor optimized by measuring the temperature distribution characteristics of heating resistor according to temperature and flow rate with IR (infrared) thermography.

\section{EXPERIMENTAL}

The process sequence of the flow sensor was to form a $400 \mathrm{~nm} \mathrm{Si}_{3} \mathrm{~N}_{4}$ thin film at $850{ }^{\circ} \mathrm{C}$ on a 6-inch p-type silicon substrate using LPCVD. A Pt pattern having a length of $3030 \mu \mathrm{m}$ was formed on the upper and lower sides of the Pt heater. The heating part and the sensing part were formed of a Ti $(20 \mathrm{~nm}) / \mathrm{Pt}(200 \mathrm{~nm})$ thin film using an E-beam evaporator. In order to protect the pattern of fabricated sensor, $\mathrm{Si}_{3} \mathrm{~N}_{4}$ passivation thin film was formed on the fabricated sensor at $350{ }^{\circ} \mathrm{C}$ by using PECVD (plasma enhanced chemical vapor deposition). In addition, the flow sensor was fabricated with membrane structure through $\mathrm{KOH}$ etching, so that the heat distribution was made efficient according to the flow rate. Also, to increase the sensitivity of the sensing part, each sensing part was composed of three pairs at the upper and lower parts with intervals of $500 \mu \mathrm{m}$ based on the central heating part. Figure 1 shows the measurement setup consist of the flow sensor and infrared thermography camera. As the temperature increased from room temperature to about $180{ }^{\circ} \mathrm{C}$, the resistance of the sensing part decreased nonlinearly to several $\mathrm{k} \Omega$. The $\mathrm{Si}_{3} \mathrm{~N}_{4}$ membrane structure formed through the $\mathrm{KOH}$ etching can be used to prevent the heat generated in the heater from being conducted to the silicon substrate. The temperature changes according to the flow rate based on the central heater and the images taken by the IR camera are shown. As the flow rate increases, the overall temperature was decreased. Therefore, the flow rate 
can be measured by using the difference between the resistance change due to the overall temperature decrease of the heater and the upper and lower sensing resistance values. Also, the measurement range of the sensor can be adjusted by modulating the position of the sensing part based on the central heating part.

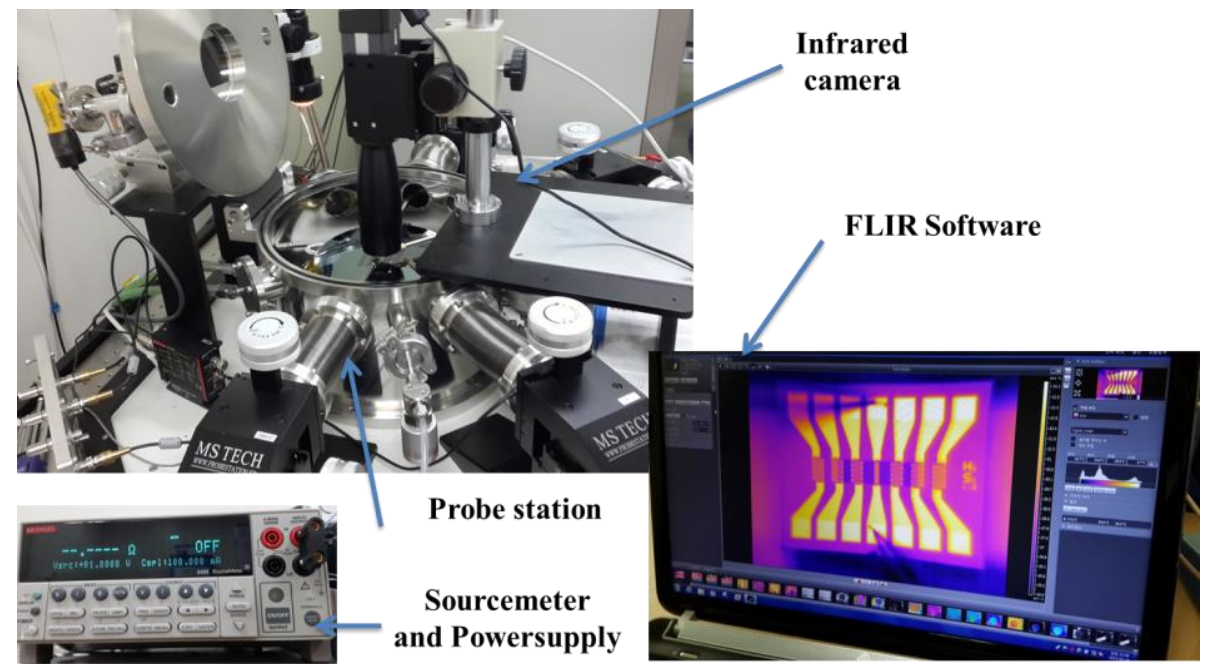

Fig. 1 The measurement setup.

\section{RESULTS AND CONCLUSIONS}

In this study, the characteristics of the flow sensor were observed using the IR camera. The linear temperature change of the central heater was presented using a-Si/Cu heating resistor. Also, it was observed that the temperature decreasing was caused by the flow rate of $0 \sim 4 \mathrm{~m} / \mathrm{s}$ and the heater cools simultaneously. In order to measure the flow rate through the cooling and the temperature difference of the heater, the membrane support of the sensor must be arranged to the upper and lower sensing parts so that the heat is not diffused through the silicon wafer. However, the temperature change occurred by the flow rate. Therefore, it is possible to measure a precise and wide range of flow rate by sensing the temperature change in the heat generating part and measuring the temperature difference cooled at the center temperature. In the future, we will propose a structure of optimized micro-sensor by adjusting pattern length and position of temperature sensor and performing precise analysis.
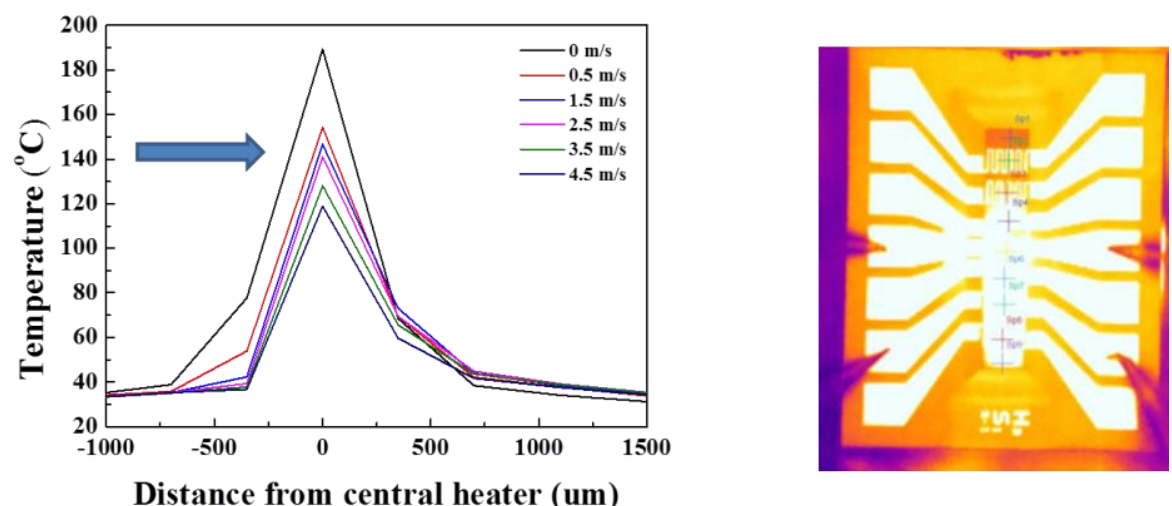

Fig.2 The temperature changes of flow sensor and infrared image according to the flow rate.

\section{ACKNOWLEDGMENT}


This work was supported under the framework of 2017 international cooperation program managed by National Research Foundation of Korea (Grant No: 2017K1A4A3013662) and WCSL (World Class Smart Lab) research grant directed by Inha University.

\section{REFERENCES}

[1] S.J. Leigh, R.J. Bradley, C.P. Pussell, D.R. Billson, D.A. Hutchins, A simple, low-cost conductive composite material for 3D printing of electronic sensors, PloS one. 7 (2012) e49365.

[2] B.C. Gross, J.L. Erkal, S.Y. Lockwood, C.P. Chen, D.M. Spence, Evaluation of 3D printing and its potential impact on biotechnology and the chemical sciences, ANALYTICAL CHEMISTRY. 86 (2014) 3240-3253.

[3] S. Bagavathiappan, B.B. Lahiri, T. Saravanan, J. Philip, T. Hayakumar, Infrared thermography for condition monitoring-a review, Infrared Physics \& Technology. 60 (2013) 35-55. 